\title{
Bioreactor Scale-Up and Kinetic Modeling of Lactic Acid and Biomass Production by Enterococcus faecalis SLT13 during Batch Culture on Hydrolyzed Cheese Whey
}

\author{
Manel Ziadi $\mathbb{D}^{1},{ }^{1}$ Sana M'Hir $\mathbb{D}^{1},{ }^{1}$ Abdelkarim Aydi, ${ }^{2}$ and Moktar Hamdi ${ }^{1}$ \\ ${ }^{1}$ Laboratory of Microbial Ecology and Technology (LETMi), The National Institute of Applied Sciences and Technology (INSAT), \\ Carthage University, 2 Boulevard de la Terre, BP 676, 1080, Tunis, Tunisia \\ ${ }^{2}$ Laboratory of Materials Molecules and Applications, Preparatory Institute for Scientific and Technical Studies, \\ Carthage University, BP 51, La Marsa, 2075, Tunis, Tunisia
}

Correspondence should be addressed to Manel Ziadi; manel_z52@yahoo.fr and Sana M'Hir; sana2617@yahoo.fr

Received 26 June 2019; Accepted 15 October 2019; Published 9 March 2020

Guest Editor: Abdullah Al Loman

Copyright (C) 2020 Manel Ziadi et al. This is an open access article distributed under the Creative Commons Attribution License, which permits unrestricted use, distribution, and reproduction in any medium, provided the original work is properly cited.

\begin{abstract}
Kinetic modeling of biomass and lactic acid production by Enterococcus faecalis SLT13 have been developed during batch culture in M17 and Hydrolyzed Cheese Whey (HCW) in $2 \mathrm{~L}$ and $20 \mathrm{~L}$ bioreactors. The specific growth rate $\mu_{\max }$ was higher in $20 \mathrm{~L}$ bioreactor $\left(1.09 \mathrm{~h}^{-1}\right)$; however, the maximum specific lactic acid production rate $q_{p}$ max and maximum specific sugar utilization rate $q_{s}$ max were higher in $2 \mathrm{~L}$ bioreactor. Biomass and sugar utilization were affected by lactic acid inhibition in HCW. No effects of substrate inhibition have been observed. Substrate limitation of biomass has been observed on HCW in $20 \mathrm{~L}$ bioreactor; the substrate limitation constant for biomass $K_{s x}$ was $4.229 \mathrm{~g} / \mathrm{L}$. Substrate limitation of sugar consumption has been observed on M17 in $2 \mathrm{~L}$ bioreactor; the substrate limitation constant for sugar consumption $K_{s s}$ was $2.73 \mathrm{~g} / \mathrm{L}$. Compared to experimental data, the model provided good predictions for biomass, sugar consumption, and lactic acid production.
\end{abstract}

\section{Introduction}

Actually, the lactic acid and its derivative polylactic acid (PLA) market appears among the most important markets of the chemical industry. This market is in clear progress and it is predictable to attain USD 3.82 billion and 5.16 billion on 2020 for the lactic acid and the PLA, respectively. This growth is due to several reasons: the increasing request for the eco-friendly products, the increasing prices of products derived from the petrochemistry, and the limited reserves of fossil energy [1].

The lactic acid presents several applications, essentially the food industries (preservative and acidulant), textile, and pharmaceutical industries. Moreover, the expansion of PLA market is the main reason for lactic acid market rising. Indeed, the lactic acid is used as monomer for PLA production [2]. For its bioavailability and biodegradability, the PLA is used not only in the food industries for packaging of sensitive foods (i.e., eggs) and tablets in the pharmaceutical industry, but also in electronics and textile, which can be substituted for synthetic polymers derived from petrochemistry.

Two processes are used to produce industrially lactic acid: chemical synthesis and microbial fermentation. However, microbial fermentation presents numerous advantages compared to chemical synthesis; these advantages are essentially environmental; fermentation is a clean process; also the lactic acid obtained by fermentation is optically pure (L (+)-lactic acid) compared to the racemic mixture (50\% L-lactic acid and 50\% D-lactic acid) obtained chemically [3]. Indeed, PLA with L (+)-lactic acid content higher than $90 \%$ is a crystalline polymer and is preferred for commercial uses than the amorphous polymer with high content of the D-isomer [4].

For advantageous commercial process, choosing an appropriate carbon source for lactic acid production is crucial. Indeed, a promising substrate must be of low cost, does not contain contaminants, and allows fast growth of the lactic acid producer microorganism as well as high 
recovery in lactic acid [5]. Thus, several substrates have been used for the production of lactic acid through microbial fermentation. Among them, cheese whey, a major biowaste of the dairy industry, is a good candidate for this purpose. This byproduct contains $5-8 \%(\mathrm{w} / \mathrm{w})$ of dry matter, in which lactose is approximately $60-80 \%$, proteins 10-20\%, minerals, vitamins, fat, lactic acid, and trace elements [6]. Cheese whey has been widely used for various productions including organic acids, single-cell proteins, enzymes, and ethanol [7].

Many microorganisms are involved in lactic acid production from varied feedstock including Lactic Acid Bacteria (LAB), yeasts, and fungi. LAB species used for lactic acid production include Lactobacillus, Streptococcus, and Enterococcus producing $\mathrm{L}(+)$-lactic acid, while Leuconostoc and Lactobacillus bulgaricus produce $\mathrm{D}(-)$-lactic acid [8]. Genetically modified Saccharomyces cerevisiae was used for the production of lactic acid in continuous fermentation [9]. Rhizopus is the major fungi used for lactic acid production; the species $R$. oryzae and $R$. microsporus have been previously used and high amounts of lactic acid were formed [10]. Others bacterial strains, Bacillus and Escherichia coli, were also used for the production of both lactate isomers [11,12].

Among lactic acid bacteria involved in lactic acid production, Enterococcus faecalis has been described in many studies as lactic acid producer. Enterococcus faecalis RKY1 is able to produce lactic acid from agricultural feedstock, such as wheat, barley, corn, hydrol, soybean curd residues, malt, and from single and mixed sugars [13-15]. The same strain was used by Nandsana and Kumar [16] to produce lactic acid from molasses. Enterococcus faecium No. 78 has been used for lactic acid production from liquefied sago starch [17]. Enterococcus faecalis QU11 is able to produce lactic acid from glycerol fermentation [18].

With increasing interest in the industrial application of lactic acid and PLA, different kinetic models have been developed in order to study the growth and lactic acid production by different species of LAB. Indeed, kinetic modeling is an essential step to optimize a fermentation process, as though models help in process control, reducing process costs and time, and improving product quality [19].

Earlier studies have described lactic acid production by Lactobacillus sp. using Luedeking-Piret model. This kind of model relates cell growth and lactic acid production by including two coefficients: growth-associated coefficient $(\alpha)$ and a non-growth-associated coefficient $(\beta)$. Unstructured kinetic models such as the Gompertz equation have been also used [20]. Further developments have proposed models which included Monod equation to describe the relation between a limiting substrate and biomass growth and then product formation [21, 22]. Monod equation relates the specific growth rate $(\mu)$ to the concentration of a single growthlimiting substrate $(S)$ via two parameters, the maximum specific growth rate $\left(\mu_{\max }\right)$ and the substrate affinity constant $\left(K_{S}\right)$. Other authors have proposed the inhibitory effects of high initial lactose concentrations, lactose limitation, and lactate inhibition [23, 24], while others developed models showing the inhibition effects of both associated and dissociated forms of lactic acid $[25,26]$.
This study is conducted to find the most suitable model that describes biomass and lactic acid production by Enterococcus faecalis SLT13 in batch cultures in a medium prepared with hydrolyzed cheese whey and in a synthetic medium (M17). The effects of culture media and scale-up from 2 to $20 \mathrm{~L}$ bioreactors on the kinetic parameters were studied.

\section{Materials and Methods}

2.1. Bacterial Strain and Culture Conditions. The strain Enterococcus faecalis SLT13 used in this work was isolated from Tunisian traditional fermented milk "Leben" [27]. Stock cultures of this strain were stored in M17 broth containing $20 \%$ glycerol (Merck) at $-80^{\circ} \mathrm{C}$.

Enterococcus faecalis cells were grown in M17 broth (Merck), containing glucose as main carbon source or in papain Hydrolyzed Cheese Whey (HCW). Cheese whey powder (BHA, Belgium SA) was rehydrated in distilled water $(6 \% \mathrm{w} / \mathrm{v})$. All fermentations were supplemented with (/L): 2 g yeast extract (YE); $0.5 \mathrm{~g} \mathrm{NH}_{4} \mathrm{Cl} ; 2 \mathrm{~g} \mathrm{~K}_{2} \mathrm{HPO}_{4}$; and $25 \mathrm{mg} \mathrm{MnSO}_{4}$. Papaïn was provided by the Walloon Center of Industrial Biology (CWBI, Belgium) and had an activity of $22000 \mathrm{IU}$. The enzyme: substrate ratio was 0.5 : $100(\mathrm{w} / \mathrm{w})$. The whey hydrolysis was carried out at $\mathrm{pH} 5.0$ and $60^{\circ} \mathrm{C}$. The enzyme was inactivated by thermal treatment $\left(90^{\circ} \mathrm{C}\right.$ for $\left.5 \mathrm{~min}\right)$ and cooled in an ice bath. Hydrolysis time was $30 \mathrm{~min}$. After hydrolysis, the $\mathrm{pH}$ was adjusted to 7.0.

Batch cultures using M17 or HCW media were carried out in $2 \mathrm{~L}$ bioreactor Biostat $\mathrm{B}$ (B. Braun Biotech International, Melsungen, Germany). A working volume of $1.5 \mathrm{~L}$ was used, which comprised $1.4 \mathrm{~L}$ growth medium and $100 \mathrm{~mL}$ of inoculum. Inoculum was prepared by adding $100 \mu \mathrm{L}$ of the preserved strain in $250 \mathrm{~mL}$ Erlenmeyer flask containing $100 \mathrm{~mL}$ of M17 broth (approx. $1 \times 10^{7} \mathrm{CFU} / \mathrm{mL}$ ) and then incubated at $30^{\circ} \mathrm{C}$. The media used were sterilized in the bioreactor at $121^{\circ} \mathrm{C}$ for $15 \mathrm{~min}$. The set point of $\mathrm{pH}$ was $7.0 \pm 0.2$, temperature $30^{\circ} \mathrm{C}$, and stirrer speed $100 \mathrm{rpm}$. The $\mathrm{pH}$ was adjusted with $1 \mathrm{~N} \mathrm{HCl}$ or $1 \mathrm{~N} \mathrm{NaOH}$. The fermentation was run for $10 \mathrm{~h}$ in M17 and $24 \mathrm{~h}$ in HCW. Samples were withdrawn every $2-4 \mathrm{~h}$ for determination of glucose, lactose, and lactic acid concentrations and viable cells count $(\mathrm{CFU} / \mathrm{mL})$.

Batch culture in $20 \mathrm{~L}$ bioreactor was conducted as follows: $100 \mu \mathrm{L}$ of the preserved strain was inoculated into $20 \mathrm{~mL}$ vial containing $10 \mathrm{~mL}$ M17 broth and then incubated at $30^{\circ} \mathrm{C}$ for $12 \mathrm{~h}$ and transferred again in $250 \mathrm{~mL}$ Erlenmeyer flask containing $200 \mathrm{~mL} \mathrm{M17} \mathrm{broth} \mathrm{(preculture} P_{1}$ ) that was incubated at $30^{\circ} \mathrm{C}$ for $16 \mathrm{~h}$. The preculture $P_{1}$ from the exponential growth phase was inoculated into $1 \mathrm{~L}$ Erlenmeyer flask containing $800 \mathrm{~mL} \mathrm{M17}$ broth (preculture $P_{2}$ ). Batch culture was performed in $20 \mathrm{~L}$ stirred bioreactor (Biolafitte, France) in HCW. The media used were sterilized in the bioreactor at $121^{\circ} \mathrm{C}$ for $15 \mathrm{~min}$. The preculture $P_{2}$ in the exponential growth phase prepared in $1 \mathrm{~L}$ Erlenmeyer flask was added to the culture medium. The temperature of the bioreactor was maintained at $30^{\circ} \mathrm{C}$ and the $\mathrm{pH}$ was controlled at $7 \pm 0.2$ by the addition of $\mathrm{NaOH}(3 \mathrm{~N})$. Samples were withdrawn every $2-4 \mathrm{~h}$ for determination of lactose and lactic acid concentrations and viable cells count $(\mathrm{CFU} / \mathrm{mL})$. All experiments were conducted in triplicate. 
2.2. Biomass Determination. For biomass determination, a calibration curve relating dry cell weight with the cell concentration (estimated by viable cells counting method) was used. For cells counting, $0.1 \mathrm{~mL}$ of diluted sample was spread over M17-agar surface. The plates were then incubated for one night at $30^{\circ} \mathrm{C}$. The number of colonies was counted and expressed in CFU/mL (Colony-Forming Units/mL).

2.3. Analytical Analysis. Glucose, lactose, and lactic acid concentrations in the fermentation broth were measured by HPLC (Agilent Technologies) using an ion-exclusion column (SupelCo Gel C-610H) operated at $35^{\circ} \mathrm{C}$. Components were eluted with $0.1 \%$ phosphoric acid at a flow rate of $0.5 \mathrm{~mL} \cdot \mathrm{min}^{-1}$. Detection was accomplished by a refractive index detector.

\subsection{Kinetic Model}

2.4.1. Model Development. The model used in this work is the one developed by Nandasana and Kumar [16] for lactic acid production by Enterococcus faecalis RKY1 and is as follows:

Specific growth rate:

$$
\mu=\frac{\mu_{\max } S K_{i X}}{\left(K_{S X}+S\right)\left(K_{i X}+S\right)} e^{-\left(P / K_{p X}\right)} .
$$

The biomass production rate:

$$
\frac{\mathrm{d} X}{\mathrm{~d} t}=\left(\mu-K_{d}\right) X .
$$

The substrate consumption rate:

$$
\frac{\mathrm{d} S}{\mathrm{~d} t}=-q_{s, \max } \frac{S K_{i S}}{\left(K_{S S}+S\right)\left(K_{i S}+S\right)} e^{-\left(P / K_{p S}\right)} X .
$$

The product production rate:

$$
\frac{\mathrm{d} P}{\mathrm{~d} t}=\alpha \frac{\mathrm{d} X}{\mathrm{~d} t}+q_{p, \max } \frac{S K_{i P}}{\left(K_{S P}+S\right)\left(K_{i P}+S\right)} e^{-\left(P / K_{p p}\right)} X .
$$

For (3), the usual assumption proposed by Nandasana and Kumar [16] was not used, due to the nature of the process; a minus sign was included. According to the same authors, these assumptions are taken in consideration for the simplification of the modeling step:

(i) $K_{s s}=K_{s p}$, low lactose concentration affects in the same way lactose uptake and lactic acid production;

(ii) $K_{i s}=K_{i p}$, high lactose concentration inhibits in the same way lactose uptake and lactic acid production;

(iii) $K_{p s}=K_{p p}$, lactate concentration affects in the same way lactose uptake and lactic acid production.

The method used to solve model differential equations for a given set of kinetic parameters was ODE113s solver of MATLAB [28], in order to obtain $X, S$, and $P$ values. The initial conditions were the experimental values of $X, S$, and $P$ at time zero $\left(t=t_{0}\right)$.

2.4.2. Model Integration. In order to estimate kinetic parameters, a search of these parameters values, for which predicted values of $X, S$, and $P$ are closer to the experimental one, needs to be applied. For this process, an optimization method was used. The nonlinear optimization method chosen for this problem was the interior-point approach to constrained minimization using the "fmincon" function in MATLAB $[29,30]$. This function is a local search algorithm that starts with an initial guess and finds a constrained minimum.

The objective function (OF) used in this minimization was in the form of

$$
\begin{aligned}
\mathrm{OF}_{X} & =\frac{\sum_{i=1}^{n}\left(X_{i}-X_{i, \mathrm{EXP}}\right)^{2}}{\sum_{i=1}^{n}\left(X_{i}-\overline{X_{i}}\right)^{2}} \cdot 100, \\
\mathrm{OF}_{S} & =\frac{\sum_{i=1}^{n}\left(S_{i}-S_{i, \mathrm{EXP}}\right)^{2}}{\sum_{i=1}^{n}\left(S_{i}-\overline{S_{i}}\right)^{2}} \cdot 100, \\
\mathrm{OF}_{P} & =\frac{\sum_{i=1}^{n}\left(P_{i}-P_{i, \mathrm{EXP}}\right)^{2}}{\sum_{i=1}^{n}\left(P_{i}-\overline{P_{i}}\right)^{2}} \cdot 100, \\
\mathrm{OF} & =\frac{\mathrm{OF}_{X}+\mathrm{OF}_{S}+\mathrm{OF}_{P}}{3} .
\end{aligned}
$$

Thus, it was considered in the estimation of parameters that all variables are equally important and given equal weight to the approximation error for each one of them. The procedure to find the parameters was repeated at least 30 times, with random initial guesses, in order to assure a good solution.

\section{Results and Discussion}

3.1. Effect of Culture Media and Scale-Up on Lactic Acid and Biomass Productivity. This study is conducted to evaluate the effect of scale-up on growth and lactic acid production by Enterococcus faecalis SLT13. The fermentation was first carried out at laboratory scale, in $2 \mathrm{~L}$ bioreactor, than at semipilot scale, in $20 \mathrm{~L}$ bioreactor. All the studied fermentations were conducted in Stirred Tank Reactor (STR). Moreover, two distinct culture media were analyzed including the synthetic medium M17 and the papain Hydrolyzed Cheese Whey (HCW) in order to characterize the growth kinetics of Enterococcus faecalis SLT13. $2 \mathrm{~L}$ bioreactor cultures were conducted in M17 and HCW under controlled conditions, while only HCW was used for $20 \mathrm{~L}$ bioreactor; the same parameters $(\mathrm{pH}$, temperature, and stirrer) were maintained.

The key kinetic parameters during the culture of $E$. faecalis in the conditions mentioned below are estimated experimentally and summarized in Table 1 .

Whereas the final biomass is higher in synthetic media (M17), lactic acid concentration is more important when E. faecalis was grown on HCW. Lactic acid yield on lactose $\left(Y_{p s}\right)$ increases significantly in HCW (from 0.314 to $0.98 \mathrm{~g} / \mathrm{g}$ ). Lactic acid yield obtained for the strain E. faecalis RKY1 based on sugar consumption varies from 0.90 to 0.99 for different initial sugar concentrations [2].

The ability of Enterococcus faecalis SLT13 to conduct lactose-to-lactic acid production is enhanced in hydrolyzed 
TABLE 1: Main experimental data from the different batch cultures of Enterococcus faecalis SLT13.

\begin{tabular}{lccc}
\hline Parameters & 2 L M17 & 2 L HW & 20 L HW \\
\hline Biomass (g/L) & 3.16 & 2.57 & 2.67 \\
Lactic acid (LA) (g/L) & 6.04 & 32.23 & 22.8 \\
$\mathrm{Y}_{p s}(\mathrm{~g} / \mathrm{g})$ & 0.314 & 0.98 & 0.72 \\
$\mathrm{Y}_{x s}(\mathrm{~g} / \mathrm{g})$ & 0.142 & 0.069 & 0.074 \\
Volumetric productivity & 0.60 & 1.33 & 0.95 \\
LA (g/L·h) & & & \\
\hline
\end{tabular}

whey. This suggests that the carbon/nitrogen ratio in this kind of culture media is more favorable to lactic acid production than biomass growth. This fact is confirmed by the decrease of biomass yield on lactose $\left(Y_{x s}\right)$, which suggests that the carbon source available in the medium is rather directed towards lactic acid production. Values of $Y_{x s}$ are in the same range of magnitude than those reported for others species: Enterococcus faecalis CBRDO1 cultivated in LA5 medium supplemented with various concentration of glucose $\left(5-20 \mathrm{~g} \cdot \mathrm{L}^{-1}\right)$ presented $Y_{x s}$ from 0.03 to $0.06 \mathrm{~g} / \mathrm{g}$ [31]. Enterococcus faecalis RKY1 grown on cane-sugar molasses had 0.07-0.19 g/g [2]. Ziadi et al. [21] presented values varying between 0.18 and $0.20 \mathrm{~g} / \mathrm{g}$ for two strains of Lactococcus lactis SLT6 and SLT10 cultured on hydrolyzed cheese whey.

The volumetric lactic acid productivities are higher in HCW and reach $1.33 \mathrm{~g} / \mathrm{L} \cdot \mathrm{h}$ in $2 \mathrm{~L}$ bioreactor. The ability of E. faecalis to produce $\mathrm{L}(+)$ - lactic acid from glucose or lactose by homolactic fermentation was earlier demonstrated in the literature. Lactate is generated through pyruvate reduction and two enzymes are involved in this reaction; the cytosolic NAD+-dependent $\mathrm{L}-(+)$-lactate dehydrogenases: ldh-1 and ldh-2 [32].

Enterococcus faecalis is able to produce lactic acid from different substrates; Oh et al. [15] reported productivities higher than $0.8 \mathrm{~g} / \mathrm{L} \cdot \mathrm{h}$ for E. faecalis RKY1 using barley and wheat as nutrient source.

While biomass is enhanced by scale-up, lactic acid production is negatively affected although the scale-up ratio $1: 10$ is respected. This decrease can be explained by many reasons. During industrial fermentation, scale-up causes changes in the geometry and consequently hydrodynamic of the bioreactor, especially for the STR. Two important changes occurred and can affect microbial survival conducting to a decrease of productivities: the mass transfer and the shear stress. The changed geometric conditions affect mixing behavior and consequently mass transfer which lead to a less availability of nutriments for the cells. The increasing shear stress during scale-up affects intrinsic resistance of the lactic-acid-producer microorganism [33].

Indeed, in mechanical agitated bioreactors and independently from microorganism type, the stirrer is the main dispersing tool allowing contact between both phases, biotic and abiotic, of the system. For an optimal process with optimal kinetic parameters, mass transfer between cells and culture media is crucial. Thus, choosing the appropriate stirrer speed and design is essential [34]. Several stirrer designs are available; the most used on microbial fermentation is the flat blade turbine type Rushton (containing 4,6 , or 8 blades) assuring a radial flow. This type of turbine is adapted to the agitation of Newtonian fluids such as culture media. However, shear stress caused by this type of turbine is important, particularly in the case of fragile microorganisms. Knowing that the impeller used in both $2 \mathrm{~L}$ and $20 \mathrm{~L}$ bioreactors consists of 2 and 3 turbine type Rushton, respectively, the decrease in lactic acid productivity during scale-up can be attributed to the high shear stress caused by this kind of turbine. The effect of shear stress on LAB was earlier demonstrated when studying the cell metabolism of Lactobacillus delbrueckii subsp. bulgaricus; cells cultivated at $72 \mathrm{~Pa}$ were affected by shearing forces; however, when cultivated at $36 \mathrm{~Pa}$, metabolism was improved [35].

Thus, a good comprehension of kinetic behavior during bioreactor scale-up of lactic acid production is of crucial importance.

3.2. Estimation of Parameters. The experimental data obtained from batch fermentations of Enterococcus faecalis SLT13 on M17 and Hydrolyzed Cheese Whey in $2 \mathrm{~L}$ and $20 \mathrm{~L}$ bioreactors were used for developing the kinetic models and estimation of kinetic parameters. Results are presented in Table 2.

Differences in the maximum specific growth rate $\left(\mu_{\max }\right)$ values obtained from the parameters estimation are observed. Higher growth rate is achieved when the strain is cultivated in HCW in $20 \mathrm{~L}$ bioreactor $\left(1.09 \mathrm{~h}^{-1}\right)$. Similar values have been reported for Enterococcus faecalis RKY1 grown on cane-sugar molasses $1.6 \mathrm{~h}^{-1}$ [16]. The low growth rate in M17 was found previously for Enterococcus faecalis EF37 grown on the same medium [20]. Enterococcus faecalis CBRDO1 grown on LA5 medium supplemented with various concentration of glucose (5-20 g. $\mathrm{L}^{-1}$ ) showed $\mu_{\max }$ values of $0.59-0.64 \mathrm{~h}^{-1}$ [31].

The substrate limitation constants $K_{s x}, K_{s s}$ and $K_{s p}$ (Monod constants) for biomass production, sugar utilization and lactic acid production respectively are estimated. Although no effects of substrate limitation are observed for fermentation in HCW in $2 \mathrm{~L}$ bioreactor, substrate limitation of biomass is observed when Enterococcus faecalis SLT13 was cultured on HCW in $20 \mathrm{~L}$ bioreactor (the substrate limitation constant for growth of biomass $K_{s x}$ is $4.229 \mathrm{~g} / \mathrm{L}$ ). Substrate limitation of sugar consumption is observed when the strain is cultured on M17 in $2 \mathrm{~L}$ bioreactor (the substrate limitation constant for sugar consumption $K_{s s}$ was $2.73 \mathrm{~g} / \mathrm{L}$ ).

In this study, substrate inhibition is not observed; the key constants for substrate inhibition $\left(K_{i x}, K_{i s}\right.$ and $\left.K_{i p}\right)$ are $\geq 100 \mathrm{~g} / \mathrm{L}$ (Table 2). Meanwhile, initial substrate concentration does not exceed $45 \mathrm{~g} / \mathrm{L}$.

The product inhibition key constants $\left(K_{p x}, K_{p s}\right.$ and $\left.K_{p p}\right)$ are also estimated and listed in Table 2. Although no substrate inhibition is observed, lactate inhibition of biomass growth occurs in HCW since the values of $K_{p x}$ are 3.77 and $5.00 \mathrm{~g} / \mathrm{L}$ for $2 \mathrm{~L}$ and $20 \mathrm{~L}$ bioreactors respectively. The values of $K_{p x}, \mathrm{~K}_{p p}$ and $K_{p s}$ reported for Enterococcus faecalis RKY1, cultured in molasses, were 17.074 and $29.1664 \mathrm{~g} / \mathrm{L}$ respectively [16]. The inhibition by lactic acid can be caused by both undissociated and dissociated forms. Seen that lactic acid is completely dissociated at $\mathrm{pH} \geq 6$ and the fermentations occurs at $\mathrm{pH} 7$, product inhibition is mainly due to completely dissociated form. 
TABLE 2: Best-fitting values of kinetic parameters obtained by modeling Enterococcus faecalis SLT13 growth, sugar utilization, and lactic acid production in M17 and HCW in $2 \mathrm{~L}$ and $20 \mathrm{~L}$ bioreactors.

\begin{tabular}{|c|c|c|c|}
\hline Kinetic parameters & 2L M17 & $2 \mathrm{~L} \mathrm{HCW}$ & $20 \mathrm{~L} \mathrm{HCW}$ \\
\hline \multicolumn{4}{|c|}{ Biomass production model } \\
\hline$\mu_{\max }\left(\mathrm{h}^{-1}\right)$ & 0.34 & 0.99 & 1.09 \\
\hline$K_{i x}(\mathrm{~g} / \mathrm{g})$ & 114.06 & 399.75 & 394.20 \\
\hline$K_{s x}(\mathrm{~g} / \mathrm{g})$ & 0.023 & 0.0023 & 4.229 \\
\hline$K_{p x}(\mathrm{~g} / \mathrm{g})$ & 18.11 & 3.77 & 5.001 \\
\hline$K_{d}\left(\mathrm{~h}^{-1}\right)$ & 0.013 & 0.0001 & 0.0001 \\
\hline \multicolumn{4}{|c|}{ Sugar utilization model } \\
\hline$K_{i s}(\mathrm{~g} / \mathrm{g})$ & 290.13 & 399.99 & 143.391 \\
\hline$K_{s s}(\mathrm{~g} / \mathrm{g})$ & 2.73 & 0.01 & 0.15 \\
\hline$K_{p s}(\mathrm{~g} / \mathrm{g})$ & 10.44 & 11.16 & 20.07 \\
\hline$q_{s \max }(\mathrm{g} / \mathrm{g} \cdot \mathrm{h})$ & 4.92 & 4.99 & 4.16 \\
\hline \multicolumn{4}{|c|}{ Lactic acid production model } \\
\hline$K_{i p}(\mathrm{~g} / \mathrm{g})$ & 135.89 & 100.00 & 373.89 \\
\hline$K_{p p}(\mathrm{~g} / \mathrm{g})$ & 5.31 & 44.99 & 42.83 \\
\hline$K_{s p}(\mathrm{~g} / \mathrm{g})$ & 0.025 & 0.01 & 0.065 \\
\hline$q_{p \max }(\mathrm{g} / \mathrm{g} \cdot \mathrm{h})$ & 1.027 & 2.04 & 1.863 \\
\hline$\alpha(\mathrm{g} / \mathrm{g})$ & 0.052 & 0.01 & 0.017 \\
\hline$\overline{R^{2}}$ & 94.34 & 88.81 & 97.87 \\
\hline
\end{tabular}

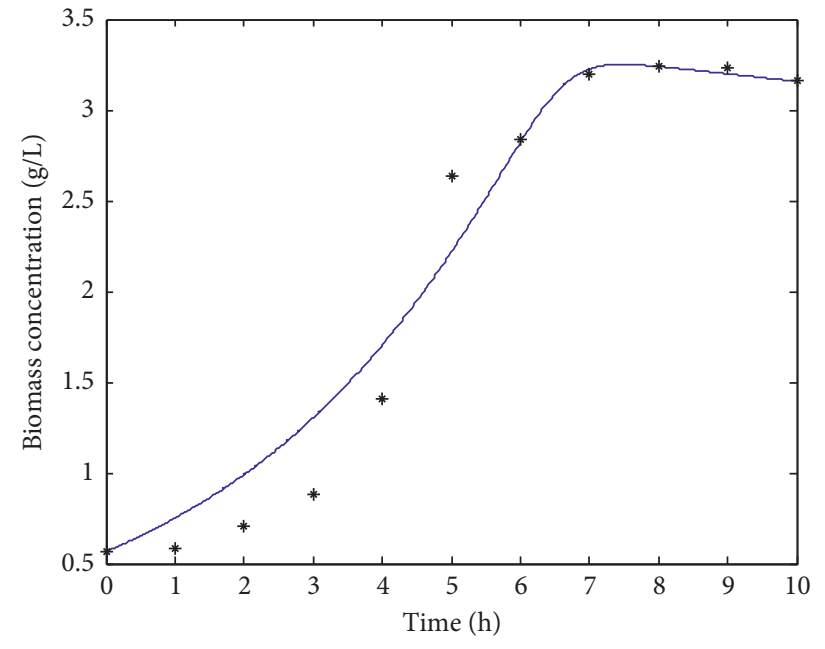

(a)

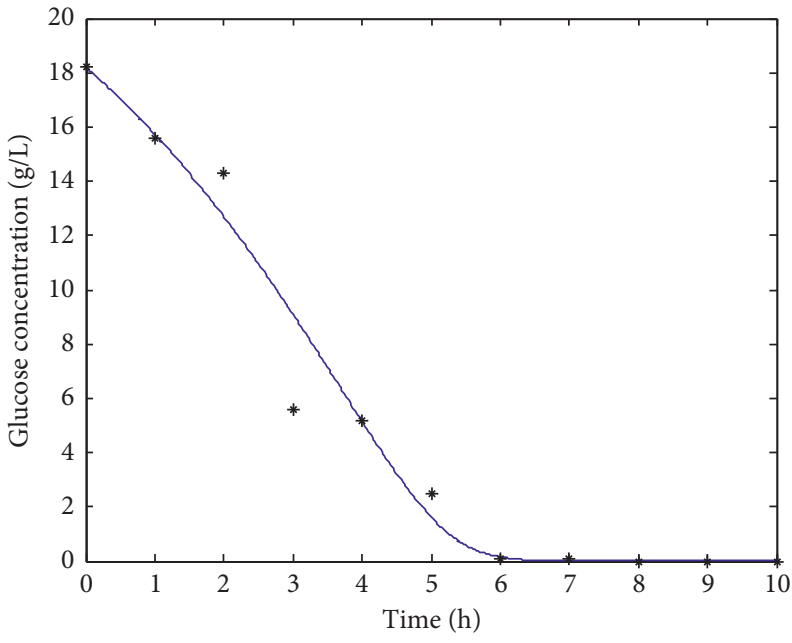

(b)

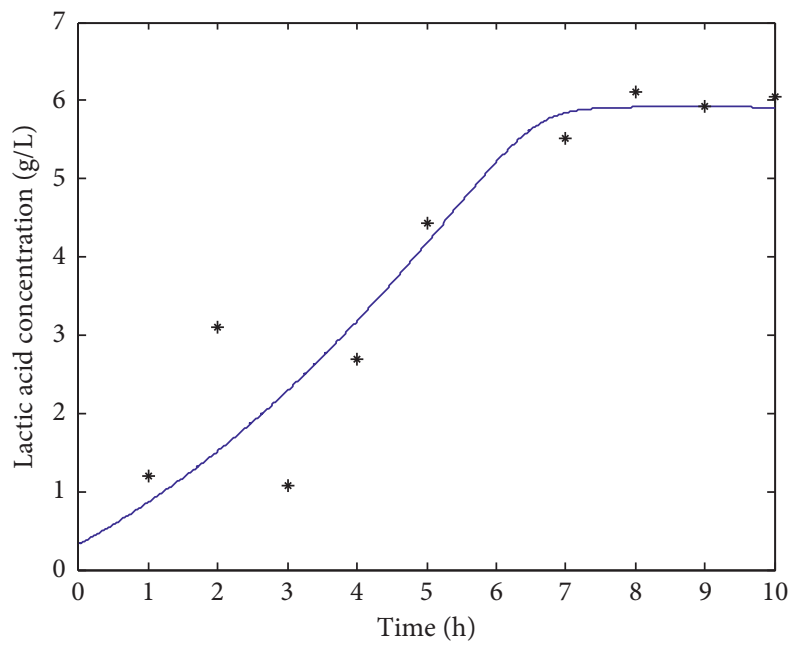

(c)

Figure 1: Experimental data (points) and simulation (lines) of biomass (a), glucose (b), and lactic acid (c) concentrations of $2 \mathrm{~L}$ bioreactor batch culture of Enterococcus faecalis SLT13 on M17 medium. 


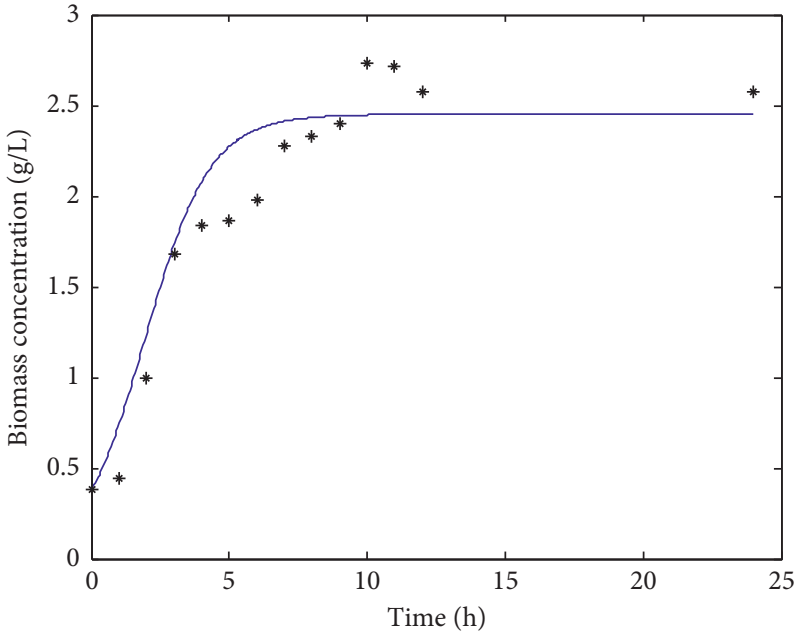

(a)

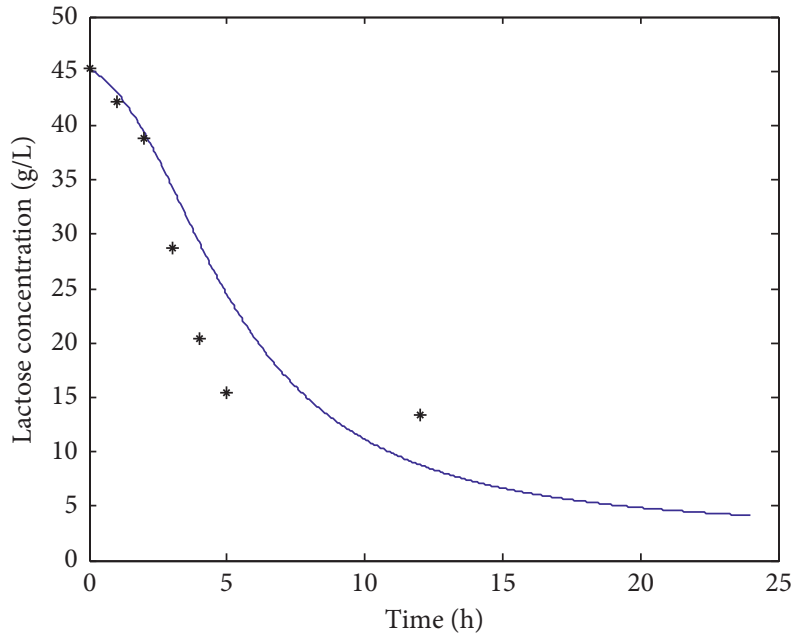

(b)

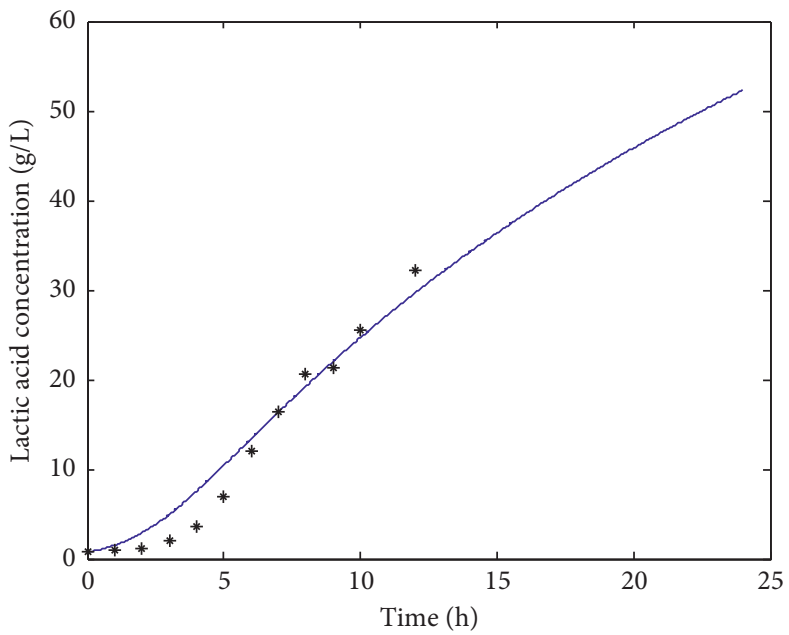

(c)

Figure 2: Experimental data (points) and simulation (lines) of biomass (a), lactose (b), and lactic acid (c) concentrations of $2 \mathrm{~L}$ bioreactor batch culture of Enterococcus faecalis SLT13 on hydrolyzed cheese whey.

For sugar consumption, the higher maximum sugar uptake rate $\left(q_{s} \max \right)$ is estimated to be $4.99 \mathrm{~g} /(\mathrm{g} \cdot \mathrm{h})$ for HCW in $2 \mathrm{~L}$ bioreactor, in spite of the values are close for the three fermentations. The maximum specific lactic acid production rate $\left(q_{p} \max \right)$ is higher for HCW and reach $2.04 \mathrm{~g} /(\mathrm{g} \cdot \mathrm{h})$ in $2 \mathrm{~L}$ bioreactor.

The growth-associated term $(\alpha)$ is very low for all the fermentations. In spite of that lactic acid production is both growth-associated and non-growth-associated and seen the value of $\alpha$, the production of lactic acid is essentially occurred in the stationary phase. Higher growth-associated parameter $(\alpha)$ was reported by Nandasana and Kumar [16] for Enterococcus faecalis molasses and was $0.26 \mathrm{~g} / \mathrm{g}$. Lactococcus lactis NZ133 grown on lactose showed a highest value $0.36 \mathrm{~g} / \mathrm{g}$ [36].

Evaluation of the cell death coefficient or endogenous decay constant $\left(K_{d}\right)$ of Enterococcus faecalis is conducted for the three fermentations. The estimated decay constants are very low and equal to $0.0001 \mathrm{~h}^{-1}$ on HCW. Nevertheless, decay constant is relatively important for the fermentation in M17 and is equal to $0.013 \mathrm{~h}^{-1}$. Death coefficient is strain dependent and different $K_{d}$ values were reported in the literature. Lactococcus lactis SLT6 and SLT10 grown in the same media used in this study (HCW) presented values of 0.093-0.26 $\mathrm{h}^{-1}$ respectively [21]. Enterococcus faecalis RKY1 grown on cane-sugar molasses had $K_{d}$ value of $0.00318 \mathrm{~h}^{-1}$ [16]. The lower $K_{d}$ value for HCW can be ascribed to the protective effect of cheese whey proteins. Indeed, whey proteins are commonly used as protective media during the spray-drying and storage of lactic acid bacteria [37].

\subsection{Comparison of Predicted and Experimental Parameters.} The model used in this study and developed by Nandasana and Kumar [16] reflects closely the kinetic behavior of Enterococcus faecalis SLT13. The comparison of experimental data and predicted one is shown in Figures 1-3.

The value of correlation coefficient $\left(R^{2}\right)$ is presented in Table 2 and is used to determine if the model fits well 


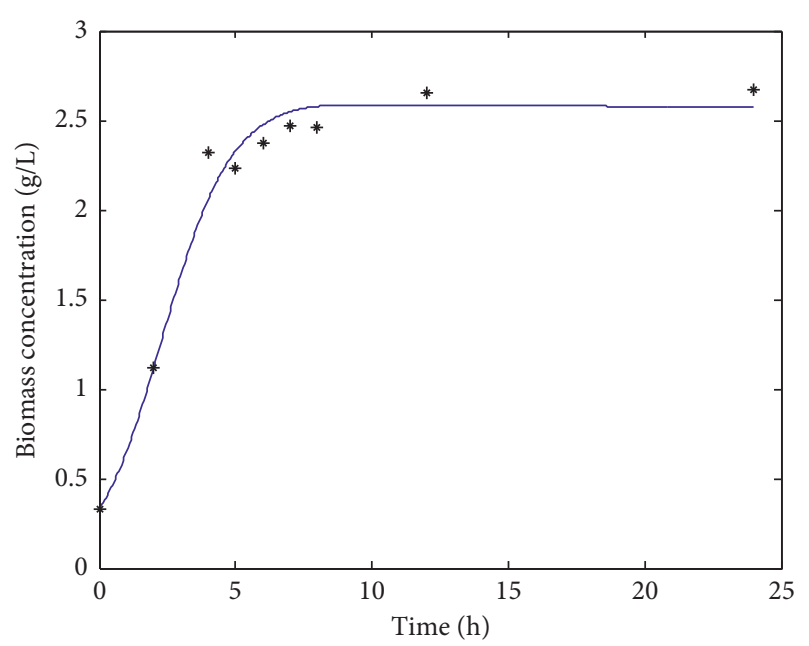

(a)

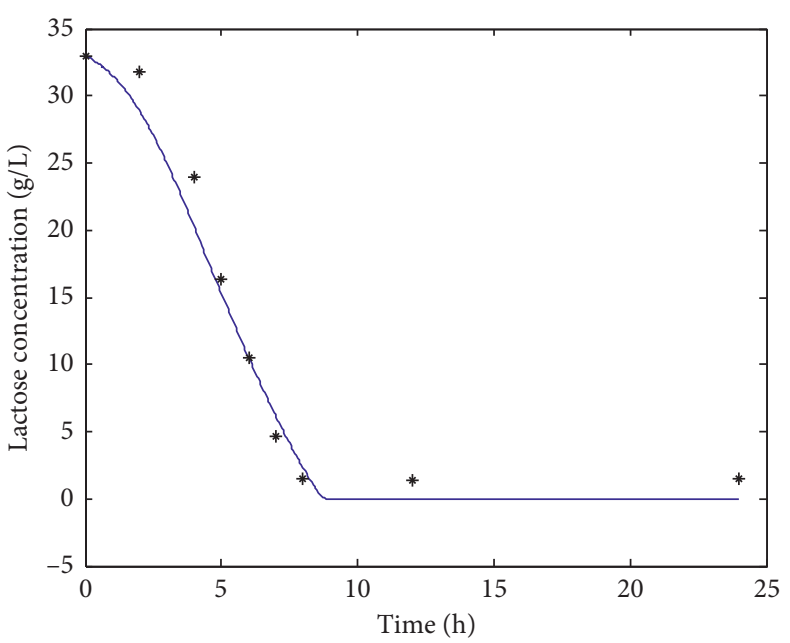

(b)

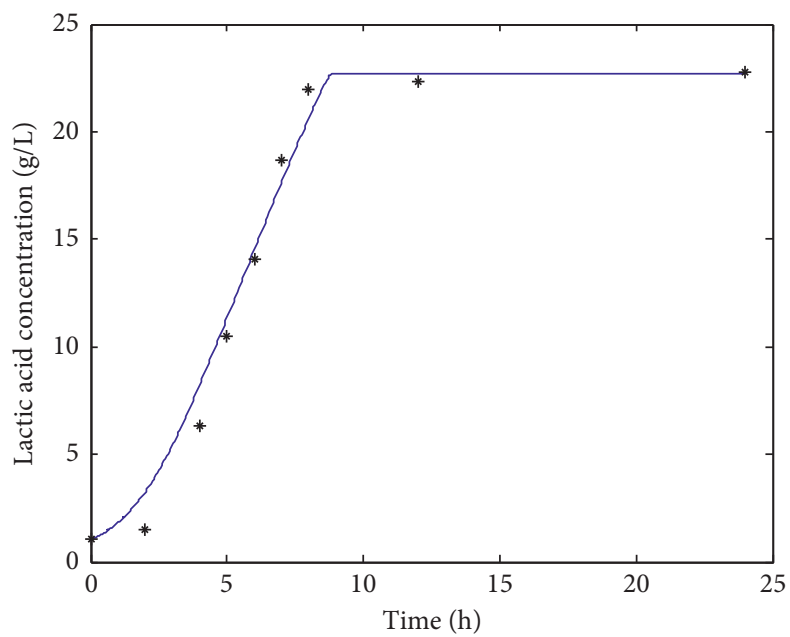

(c)

Figure 3: Experimental data (points) and simulation (lines) of biomass (a), lactose (b), and lactic acid (c) concentrations of $20 \mathrm{~L}$ bioreactor batch culture of Enterococcus faecalis SLT13 on hydrolyzed cheese whey.

the experimental data. A good linearity between model and experimental data is observed, especially for the fermentations occurring in $2 \mathrm{~L} \mathrm{M17}$ and $20 \mathrm{~L} \mathrm{HCW}$.

For all the fermentations, no lag phase is observed proving the ability of enterococci to adapt to different growth conditions. Exponential growth phase lasts $6 \mathrm{~h}$ when the strain was cultured in M17 followed by a short stationary phase. When cultured in HCW, exponential growth phase lasts only $5 \mathrm{~h}$, while the stationary phase is relatively long. Glucose is totally consumed after $6 \mathrm{~h}$ of fermentation which confirmed that in M17 medium, the main factor affecting sugar utilization is substrate limitation (the substrate limitation constant for sugar consumption $K_{s s}$ was $2.73 \mathrm{~g} / \mathrm{L}$ ). In HCW, while few quantities of lactose remain at the end of fermentation in $2 \mathrm{~L}$ bioreactor, it is almost totally consumed in $20 \mathrm{~L}$ bioreactor after about $10 \mathrm{~h}$ of fermentation $(1.4 \mathrm{~g} / \mathrm{L})$ which confirms that the growth of biomass is limited by lactose concentration since $K_{s x}$ is $4.229 \mathrm{~g} / \mathrm{L}$. Furthermore, the initial concentration of lactose is higher in $2 \mathrm{~L}$ bioreactor.
Indeed, in cheese whey powder, concentration of lactose can vary from batch to batch.

In all fermentations, lactate production began earlier in the exponential growth phase and continues during the stationary phase. Indeed, lactic acid production is growthassociated as well as non-growth-associated. Lactic acid production remains constant after $7 \mathrm{~h}$ and $10 \mathrm{~h}$ of fermentation in M17 and in HCW in $20 \mathrm{~L}$ bioreactor, respectively. However, on $\mathrm{HCW}$ in $2 \mathrm{~L}$ bioreactor, lactate concentration continues to increase with the availability of the substrate.

\section{Conclusions}

The overall results obtained in our study show that cheese whey is suitable for lactic acid production; volumetric productivities are higher than the one obtained in M17. The mathematical modeling is useful and allows characterizing growth and lactic acid production of Enterococcus faecalis SLT13 in two different media and 
two different volume bioreactors. The scale-up affects lactic acid productivity but enhances biomass growth. Substrate inhibition did not impact significantly the maximum lactic acid productivities attainable in the both $2 \mathrm{~L}$ and $20 \mathrm{~L}$ bioreactors. However, product inhibition seems to play a major role in the two studied media.

\section{Abbreviations}

$K_{d}: \quad$ Death coefficient $\left(\mathrm{h}^{-1}\right)$

$K_{i p}$ : Substrate inhibition constant for lactic acid production $(\mathrm{g} / \mathrm{L})$

$K_{i s}$ : Substrate inhibition constant for sugar consumption ( $\mathrm{g} / \mathrm{L})$

$K_{i x}$ : $\quad$ Substrate inhibition constant for growth of biomass $(\mathrm{g} / \mathrm{L})$

$K_{p p}: \quad$ Product inhibition constant for lactic acid production $(\mathrm{g} / \mathrm{L})$

$K_{p s}: \quad$ Product inhibition constant for sugar consumption (g/L)

$K_{p x}: \quad$ Product inhibition constant for growth of biomass (g/L)

$K_{s p}: \quad$ Substrate limitation constant for lactic acid production $(\mathrm{g} / \mathrm{L})$

$K_{s s}$ : Substrate limitation constant for sugar consumption $(\mathrm{g} / \mathrm{L})$

$K_{s x}: \quad$ Substrate limitation constant for growth of biomass $(\mathrm{g} / \mathrm{L})$

$P: \quad$ Lactic acid concentration $(\mathrm{g} / \mathrm{L})$

$q_{p \max }:$ Maximum specific lactic acid production rate $(\mathrm{g} /(\mathrm{g} \mathrm{h}))$

$q_{\text {smax }}$ : Maximum specific sugar utilization rate $(\mathrm{g} /(\mathrm{g} \mathrm{h}))$

$S: \quad$ Sugar concentration $(\mathrm{g} / \mathrm{L})$

$X: \quad$ Biomass concentration $(\mathrm{g} / \mathrm{L})$

$Y_{p / x}: \quad$ Lactic acid yield on growth of biomass $(\mathrm{g} / \mathrm{g}$ )

$Y_{p / s}: \quad$ Lactic acid yield on sugar consumption (g/g)

$Y_{x / s}:$ Biomass yield on sugar consumption $(\mathrm{g} / \mathrm{g})$.

\section{Greek Symbols}

$\alpha$ : Growth-associated constant in Luedeking-Piret model $(\mathrm{g} / \mathrm{g})$

$\mu_{\max }$ : Maximum specific growth rate $\left(\mathrm{h}^{-1}\right)$ of lactic acid

$\mu$ : $\quad$ Specific growth rate $\left(\mathrm{h}^{-1}\right)$.

\section{Data Availability}

All the numerical data used to support the findings of this study are available from the corresponding author upon reasonable request.

\section{Additional Points}

Kinetic modeling of lactic acid and biomass production by Enterococcus faecalis SLT13 was realized on synthetic media and Hydrolyzed Cheese Whey. Hydrolyzed Cheese Whey enhances lactic acid production. Scale-up from $2 \mathrm{~L}$ bioreactor to $20 \mathrm{~L}$ bioreactor increases biomass but not lactic acid production. Biomass growth and sugar utilization are affected by lactic acid inhibition in $\mathrm{Hy}$ drolyzed Cheese Whey.

\section{Conflicts of Interest}

The authors declare no financial or commercial conflicts of interest regarding the publication of this paper.

\section{Acknowledgments}

This work was supported by the Ministry of Higher Education and Scientific Research (Tunisia) and the Walloon Region (Belgium) during the project: Technological Platform for the Development of Bioindustries.

\section{Supplementary Materials}

Graphical abstract representing lactic acid production by Enterococcus faecalis SLT13 during batch culture on Hydrolyzed Cheese Whey in $2 \mathrm{~L}$ and $20 \mathrm{~L}$ bioreactors. (Supplementary Materials)

\section{References}

[1] Markets and Markets, "Lactic acid market by application (biodegradable polymer, food \& beverage, personal care \& pharmaceutical) \& polylactic acid market by application (packaging, agriculture, automobile, electronics, textile), \& by geography-global trends \& forecasts to 2020," Report Code: FB 2647, Markets and Markets, Hadapsar, Pune, 2015.

[2] B. Gupta, N. Revagade, and J. Hilborn, "Poly(lactic acid) fiber: an overview," Progress in Polymer Science, vol. 32, no. 4, pp. 455-482, 2007.

[3] N. Narayanan, P. K. Roychoudhury, and A. Srivastava, "L(+) lactic acid fermentation and its product polymerization," Electronic Journal of Biotechnology, vol. 7, no. 2, 2004.

[4] R. Mehta, V. Kumar, H. Bhunia, and S. N. Upadhyay, "Synthesis of poly(lactic acid): a review," Journal of Macromolecular Science, Part C: Polymer Reviews, vol. 45, no. 4, pp. 325-349, 2005.

[5] M. A. Randhawa, A. Ahmed, and K. Akram, "Optimization of lactic acid production from cheap raw material: sugarcane molasses," Pakistan Journal of Botany, vol. 44, pp. 333-338, 2012.

[6] A.-N. Vamvakaki, I. Kandarakis, S. Kaminarides, M. Komaitis, and S. Papanikolaou, "Cheese whey as a renewable substrate for microbial lipid and biomass production by zygomycetes," Engineering in Life Sciences, vol. 10, no. 4, pp. $348-360,2010$.

[7] M. I. G. Gonzalez, "The biotechnological utilization of cheese whey: a review," Bioresource Technology, vol. 57, pp. 1-11, 1996.

[8] Y. H. Park, K. M. Cho, H. W. Kim, and C. Kim, "Method for producing lactic acid with high concentration and high yield using lactic acid bacteria," CJ CheilJedang Corporation, Seoul, Republic of Korea, U.S. Patent 7682814 B2, 2010.

[9] T. Mimitsuka, K. Sawai, K. Kobayashi et al., "Production of $\mathrm{d}$-lactic acid in a continuous membrane integrated fermentation reactor by genetically modified Saccharomyces cerevisiae: enhancement in d-lactic acid carbon yield," Journal of Bioscience and Bioengineering, vol. 119, no. 1, pp. 65-71, 2015.

[10] V. Kitpreechavanich, T. Maneeboon, Y. Kayano, and K. Sakai, "Comparative characterization of 1-lactic acid-producing 
thermotolerant rhizopus fungi," Journal of Bioscience and Bioengineering, vol. 106, no. 6, pp. 541-546, 2008.

[11] J. Qin, B. Zhao, X. Wang et al., "Non-sterilized fermentative production of polymer-grade L-lactic acid by a newly isolated thermophilic strain Bacillus sp. 2-6," PLoS One, vol. 4, no. 2, Article ID e4359, 2009.

[12] S. Zhou, T. B. Causey, A. Hasona, K. T. Shanmugam, and L. O. Ingram, "Production of optically pure D-lactic acid in mineral salts medium by metabolically engineered Escherichia coli W3110," Applied and Environmental Microbiology, vol. 69, no. 1, pp. 399-407, 2003.

[13] L. V. Reddy, Y. M. Kim, J. S. Yun, H. W. Ryu, and Y. J. Wee, "L-lactic acid production by combined utilization of agricultural bioresources as renewable and economical substrates through batch and repeated-batch fermentation of Enterococcus faecalis RKY1," Bioresource Technology, vol. 209, pp. 187-194, 2015.

[14] J. Vijayakumar, R. Aravindan, and T. Viruthagiri, "Recent trends in the production, purification and application of lactic acid," Chemical and Biochemical Engineering Quarterly, vol. 22, pp. 245-264, 2008.

[15] H. Oh, Y.-J. Wee, J.-S. Yun, S. Ho Han, S. Jung, and H.-W. Ryu, "Lactic acid production from agricultural resources as cheap raw materials," Bioresource Technology, vol. 96, no. 13, pp. 1492-1498, 2005.

[16] A. D. Nandasana and S. Kumar, "Kinetic modeling of lactic acid production from molasses using Enterococcus faecalis RKY1," Biochemical Engineering Journal, vol. 38, no. 3, pp. 277-284, 2008.

[17] K. Shibata, D. M. Flores, G. Kobayashi, and K. Sonomoto, "Direct 1-lactic acid fermentation with sago starch by a novel amylolytic lactic acid bacterium, Enterococcus faecium," Enzyme and Microbial Technology, vol. 41, no. 1-2, pp. 149-155, 2007.

[18] N. Murakami, M. Oba, M. Iwamoto et al., "L-lactic acid production from glycerol coupled with acetic acid metabolism by Enterococcus faecalis without carbon loss," Journal of Bioscience and Bioengineering, vol. 121, no. 1, pp. 89-95, 2016.

[19] J. M. Dodic, D. G. Vucurovic, S. N. Dodic, J. A. Grahovac, S. D. Popov, and N. M. Nedeljkovic, "Kinetic modeling of batch ethanol production from sugar beet raw juice," Applied Energy, vol. 99, pp. 192-197, 2012.

[20] F. Gardini, M. Martuscelli, M. C. Caruso et al., "Effects of pH, temperature and $\mathrm{NaCl}$ concentration on the growth kinetics, proteolytic activity and biogenic amine production of Enterococcus faecalis," International Journal of Food Microbiology, vol. 64, no. 1-2, pp. 105-117, 2001

[21] M. Ziadi, F. Rezouga, H. Bouallagui et al., "Kinetic study of Lactococcus lactis strains (SLT6 and SLT10) growth on papain-hydrolysed whey," World Journal of Microbiology and Biotechnology, vol. 26, no. 12, pp. 2223-2230, 2010.

[22] F. Leroy and L. De Vuyst, "Growth of the bacteriocin-producing Lactobacillus sakei strain CTC 494 in MRS broth is strongly reduced due to nutrient exhaustion: a nutrient depletion model for the growth of lactic acid bacteria," Applied and Environmental Microbiology, vol. 67, no. 10, pp. 44074413, 2001.

[23] A. W. Schepers, J. Thibault, and C. Lacroix, "Lactobacillus helveticus growth and lactic acid production during $\mathrm{pH}$ controlled batch cultures in whey permeate/yeast extract medium. Part II: kinetic modeling and model validation," Enzyme and Microbial Technology, vol. 30, pp. 187-194, 2002.

[24] M. M. Alvarez, E. J. Aguirre-Ezkauriatza, A. RamírezMedrano, and Á. Rodríguez-Sánchez, "Kinetic analysis and mathematical modeling of growth and lactic acid production of Lactobacillus casei var. rhamnosus in milk whey," Journal of Dairy Science, vol. 93, no. 12, pp. 5552-5560, 2010.

[25] C. Akerberg, K. Hofvendahl, G. Zacchi, and B. HahnHägerdal, "Modelling the influence of $\mathrm{pH}$, temperature, glucose and lactic acid concentrations on the kinetics of lactic acid production by Lactococcus lactis ssp. lactis ATCC 19435 in whole-wheat flour," Applied Microbiology and Biotechnology, vol. 49, no. 6, pp. 682-690, 1998.

[26] C. J. Gadgil and K. V. Venkatesh, "Structured model for batch culture growth of Lactobacillus bulgaricus," Journal of Chemical Technology \& Biotechnology, vol. 68, no. 1, pp. 8993, 1997.

[27] M. Ziadi, S. Mhir, R. Dubois-Dauphin et al., "Analysis of volatile compounds, amino acid catabolism and some technological properties of Enterococcus faecalis strain SLT13 isolated from artisanal Tunisian fermented milk," British Microbiology Research Journal, vol. 14, no. 1, pp. 1-12, 2016.

[28] L. F. Shampine and M. K. Gordon, Computer Solution of Ordinary Differential Equations: The Initial Value Problem, W. H. Freeman and Company, San Francisco, CA, USA, 1975.

[29] R. H. Byrd, J. C. Gilbert, and J. Nocedal, "A trust region method based on interior point techniques for nonlinear programming," Mathematical Programming, vol. 89, no. 1, pp. 149-185, 2000.

[30] R. H. Byrd, M. E. Hribar, and J. Nocedal, "An interior point algorithm for large-scale nonlinear programming," SIAM Journal on Optimization, vol. 9, no. 4, pp. 877-900, 1999.

[31] M. R. Subramanian, S. Talluri, and L. P. Christopher, "Production of lactic acid using a new homofermentative Enterococcus faecalis isolate," Microbial Biotechnology, vol. 8, no. 2, pp. 221-229, 2015.

[32] A. Feldman-Salit, S. Hering, H. L. Messiha et al., "Regulation of the activity of lactate dehydrogenases from four lactic acid bacteria," Journal of Biological Chemistry, vol. 288, no. 29, pp. 21295-21306, 2013.

[33] F. R. Schmidt, "Optimization and scale up of industrial fermentation processes," Applied Microbiology and Biotechnology, vol. 68, no. 4, pp. 425-435, 2005.

[34] F. Garcia-Ochoa and E. Gomez, "Bioreactor scale-up and oxygen transfer rate in microbial processes: an overview," Biotechnology Advances, vol. 27, no. 2, pp. 153-176, 2009.

[35] J. P. Arnaud, C. Lacroix, C. Foussereau, and L. Choplin, "Shear stress effects on growth and activity of Lactobacillus delbrueckii subsp. bulgaricus," Journal of Biotechnology, vol. 29, no. 1-2, pp. 157-175, 1993.

[36] M. Boonmee, N. Leksawasdi, W. Bridge, and P. L. Rogers, "Batch and continuous culture of Lactococcus lactis NZ133: experimental data and model development," Biochemical Engineering Journal, vol. 14, no. 2, pp. 127-135, 2003.

[37] A. Ghandi, I. Powell, M. Broome, and B. Adhikari, "Survival, fermentation activity and storage stability of spray dried Lactococcus lactis produced via different atomization regimes," Journal of Food Engineering, vol. 115, pp. 83-90, 2012. 